\title{
Generalization of a Theorem by Schroer and Jost
}

\author{
F. CsIKor and G. Pócsik \\ Institute for Theoretical Physics, \\ Roland Eötvös University, Budapest
}

Received June 20, 1967

\begin{abstract}
It is proved that a hermitian scalar local field satisfying the Wightman axioms is a free one if a single $n$-point Wightman function $(n \neq 1, n \neq 3)$ satisfies the homogeneous Klein-Gordon equation at least in one variable.
\end{abstract}

1. A few years ago JosT has proved the following theorem [1]: If $A(x)$ is a hermitian local scalar Wightman field with vacuum as a cyclic vector, and if for the twofold Wightman function $\left(A_{1} A_{2}\right)_{0}=\left(A_{1}{ }^{0} A_{2}{ }^{0}\right)_{0}$, where $A^{0}(x)$ is a free field of mass $m, A_{1} \equiv A\left(x_{1}\right)$ etc., then $A(x)$ is also a free field with mass $m$. The same result has been obtained by Schroer utilizing also the LSZ asymptotic condition [2], while FEDERBUSH and JoHNSon [3], in their proof, assumed an equal-time commutation relation for the field $A(x)$. The latter authors have pointed out the possibility of a simple generalization (still in the mixed canonical and Wightman framework): if $\left(A_{1} \ldots A_{2 n}\right)_{0}=\left(A_{1}{ }^{0} \ldots A_{2 n}^{0}\right)_{0}$, then $A$ is a free field $(n \geqq 1)$.

The aim of the present note is to prove a generalization of Schroer and Jost's theorem in pure Wightman framework. We shall show that $A(x)$ is a free field if the $n$-fold Wightman function $(n \geqq 2 ; n \neq 3)$ satisfies the homogeneous Klein-Gordon equation at least in one variable. This evidently holds, if one field operator is replaced by a free field in the Wightman function.

2. Let us denote by $A(x)$ a hermitian scalar local field satisfying all the Wightman axioms [4] and assume $A(x)$ has the vacuum $\psi_{0}$ as a cyclic vector. If for all $x_{j}$ 's

$$
K_{i}\left(A_{1} \ldots A_{i} \ldots A_{n}\right)_{0}=0, \quad K=\square+m^{2}
$$

for fixed $n, n \geqq 2, n \neq 3$, then $A(x) / \sqrt{c}$ is a free field satisfying

$$
K A(x)=0, \quad[A(x), A(y)]=i c \Delta(x-y ; m),
$$

$\Delta$ is the usual commutator distribution. The positive constant $c$ is specified below. In certain cases $c=1$ automatically holds [5]. 
In case of $n=2 k(k \neq 0)$, because of [1] the source $j=K A$ has the property $\left\|j(f)^{k} \psi_{0}\right\|=0$ for all test functions, that is $j(f)^{k} \psi_{0}=0$. Hence $j(f) \psi_{0}=0$. Locality, analyticity properties and cyclic vacuum demand in the usual way $j(f)=0$ for all $f[4]$. For $n=2 k+1(k>1)$ we make use of the linked cluster decomposition [4]

$$
\begin{aligned}
\lim \left(A_{1} \ldots A_{i} \ldots A_{2 k+1}\right)_{0} & \\
& =\left(A_{s_{1}} \ldots A_{s_{i}} \ldots A_{s_{2 l}}\right)_{0}\left(A_{s_{2 l+1}} \ldots A_{s_{2 k+1}}\right)_{0} ; A_{s_{i}} \equiv A_{i}
\end{aligned}
$$

if $x_{s_{2 l+1}}, \ldots, x_{s_{2 k+1}}$ are shifted in a spacelike direction to infinity. In consequence of (1) and (3) it follows

$$
K_{s_{i}}\left(A_{s_{1}} \ldots A_{s_{i}} \ldots A_{s_{2 l}}\right)_{0}=0 .
$$

Hence we again arrive at $j(f)=0^{1}$. Evidently this proof does not apply to $n=3$.

The existence of an asymptotic field, or canonical quantization would imply that $A$ possesses the commutation properties of a free field of mass $m$. It is, however, possible to complete the proof without making use of such assumptions. Namely, the Wightman functions have Fourier transforms as tempered distributions and these are different from zero only in the forward light cone. Therefore one may write

$$
(A(x) A(y))_{0}=\frac{1}{(2 \pi)^{4}} \int \varrho\left(p^{2}\right) e^{-i p \cdot(x-y)} d^{4} p
$$

and $\varrho\left(p^{2}\right) \neq 0$ only for $p^{0}>0, p^{2}>0$. Eq. (5) and $K A=0$ yield $\varrho\left(p^{2}\right)$ $=c \delta\left(p^{2}-m^{2}\right)$ for $p^{0}>0$. Thus we have

$$
(A(x) A(y))_{0}=c\left(-i \Delta^{+}(x-y ; m)\right) .
$$

Taking the norm of $A(f) \psi_{0}$, eq. (6) shows that the constant $c$ is positive. Now, we can apply Jost's theorem for $A(x) / \sqrt{c}$ concluding that it is a free field with mass $m$.

\section{References}

1. Jost, R.: Lecture. Lectures on field theory and the many-body problem. New York: Academic Press 1961.

2. Schrokr, B.: Diplomarbeit, Hamburg 1959.

3. Federbush, P. G., and K. A. Johnson: Phys. Rev. 120, 1926 (1960).

4. Streater, R. F., and A. S. Wightman: PCT, spin and statistics and all that. New York: W. A. Benjamin, Inc. 1964.

5. Csikor, F., and G. Pocsik: ITP-Budapest Report No. 221 (1967).

1 We thank Prof. R. HaAg for pointing out the possibility of extending the proof for odd $n$. 